\title{
Nonlinear optical waveguide structure for sensor application: TM case
}

\author{
H.M. Khalil ${ }^{1}$, M.M. Shabat ${ }^{2,3}$, S.Taya ${ }^{3}$, and M.M.Abadla ${ }^{4}$ \\ ${ }^{1}$ Department of Physics, College of Women's, Ain Shams \\ University, Heliopolise, Cairo, Egypt \\ 2 Max-Planck-Institute für Physik komplexer Systeme, \\ Nöthnitzer Str. 38, 01187 Dresden, Germany \\ ${ }^{3}$ Physics Department, Islamic University of Gaza, Gaza, Gaza \\ Strip, Palestinian Authority \\ ${ }^{4}$ Physics Department, Alaqsa University, Gaza Strip, \\ Palestinian Authority
}

In this work, we present an extensive theoretical analysis of nonlinear optical waveguide sensor. The waveguide under consideration consists of a thin dielectric film surrounded by a self focused nonlinear cladding and a linear substrate. The nonlinearity of the cladding is considered to be of Kerr-type. Both cases when the effective refractive index is greater and when it is smaller than the index of the guiding layer are discussed. The sensitivity of the effective refractive index to any change in the cladding index in evanescent optical waveguide sensor is derived for TM modes. Closed form analytical expressions and normalized charts are given to provide the conditions required for the sensor to exhibit its maximum sensitivity. The results are compared with those of the well known linear evanescent waveguide sensors.

Keywords: Nonlinear waveguides, biosensors, sensitivity analysis. 


\section{Introduction}

For the last two decades, planar optical waveguides have been studied intensively as sensor elements ${ }^{(1-4)}$. Optical sensors utilize the modification of chemical measurands to optical properties such as intensity, phase, frequency and polarization of an input optical signal. Optical chemical sensors have immunity to electromagnetic interference, have no danger of ignition, field resistant, small sized, safe when used in aggressive environments and mechanically stable. Such kinds of sensor are useful for highly sensitive analysis and monitoring of hazardous environments.

The essential part of a waveguide sensor consists of a high refractive index waveguiding film sandwiched between a substrate and the sample medium to be analyzed, sometimes called the "analyte". In most cases the substrate is a solid material, which has a refractive index lager than that of the analyte. Such a sensor is called normal symmetry sensor ${ }^{(5-8)}$. For the sake of power enhancement in the covering medium and hence sensitivity enhancement, sensors of reverse configurations were introduced ${ }^{(9-10)}$ for which the refractive index of the substrate is chosen to be less than that of the analyte. The recent introduction of reverse symmetry waveguides has resulted in high sensitivity sensors.

When a light beam is injected into a planar waveguide, the light is guided through the guiding layer by a series of total internal reflections at the core-cladding interface. A standing wave, also known as an evanescent wave, is formed at each point of total internal reflection along the propagation direction. The evanescent wave distributes a small part of the light into the covering medium to a distance comparable with the wavelength of the guided light. This evanescent wave is responsible for the sensing operation. When an analyte is placed in the evanescent field, change is observed in the absorption or phase shift of the light propagating in the 
waveguide, giving an indication of the concentration or refractive index of the analyte. This interaction between the analyte and evanescent field can be observed with spectroscopic techniques. One of the most important quantities in the field of sensing is the effective refractive index $(\mathrm{N})$ of the guided mode which is given by $\mathrm{N}=\mathrm{c} / \mathrm{v}_{\mathrm{ph}}$, where $\mathrm{c}$ is the speed of light in vacuum and $\mathrm{v}_{\mathrm{ph}}$ is the phase velocity. A change is induced in the effective refractive for any evanescent wave-analyte interaction. This is the basic integrated optical sensor effect. The effective refractive index changes can be induced by the formation of ultrathin film at the waveguide-cover interface (surface sensing) or by a change in the cladding refractive index when the measurand is homogeneously distributed in the cover (homogeneous sensing). Surface sensing has been used for the detection of ultrathin biological molecular layers of thickness much smaller than the wavelength of the guided light. Such a sensing scheme is currently the subject of keen interest in pharmaceutical applications such as immunoassays. It is also of interest in chemical sensing schemes where the optochemical transducing mechanism involves an ultrathin surface layer. Homogeneous sensing has a wide range of applications such as detection of harmful gases (methane, $\mathrm{CO}_{2}, \mathrm{SO}_{2}$ ), monitoring pollutants in water and detecting the concentrations of certain chemical in blood.

A normalized analysis ${ }^{(11)}$, for the design of evanescent wave sensors was carried out and the condition for the maximum achievable sensitivity was also derived for both linear TE and TM waveguide sensors. In this paper, we present an extensive theoretical analysis of nonlinear sensors in which the guiding film is surrounded by a linear substrate and a self-focusing Kerr type nonlinear cladding. The sensitivity of the sensor will be derived and the requirements for reaching the maximum sensitivity will be determined for 
TM modes. A comparison of our results with those of linear sensors will be presented.

\section{Theory}

Here we are primarily interested in examining the behavior of nonlinear slab waveguides in sensing applications. We have confined our attention to p-polarized waves propagating in a thin film that exhibits no nonlinearity. The covering medium has an intensity-dependent dielectric constant of the form ${ }^{(12-13)}$ :

$$
\varepsilon_{n l}=\varepsilon_{c}+\alpha^{\prime}\left|H_{y}\right|^{2}
$$

where $\alpha^{\prime}$ is the nonlinearity constant and $\varepsilon_{\mathrm{c}}$ is the linear part of the permittivity. We will restrict our study to the self focusing nonlinearity $\left(\alpha^{\prime}>0\right)$.

The geometry of the nonlinear waveguide sensor is shown in Fig. 1. The guiding layer has a thickness $h$ and a dielectric constant $\varepsilon_{\mathrm{f}}$. The substrate has a dielectric constant $\varepsilon_{\mathrm{s}}$. The waves in the waveguide are propagating in the $\mathrm{x}$-direction. The dispersion relations for TM-modes are written as

$$
\begin{gathered}
\tan \left(k_{o} q_{f} h\right)=\frac{\frac{X_{s}}{a_{s}}+\frac{X_{c}}{a_{c}} \tanh C}{1-\frac{X_{s}}{a_{s}} \frac{X_{c}}{a_{c}} \tanh C}, \text { for } N^{2}<\varepsilon_{f} \\
\tanh \left(k_{o} q_{f} h\right)=-\frac{\frac{X_{s}}{a_{s}}+\frac{X_{c}}{a_{c}} \tanh C}{1+\frac{X_{s}}{a_{s}} \frac{X_{c}}{a_{c}} \tanh C}, \text { for } N^{2}>\varepsilon_{f}
\end{gathered}
$$


where $\mathrm{a}_{\mathrm{s}}$ and $\mathrm{a}_{\mathrm{c}}$ are two asymmetry parameters and $\mathrm{X}_{\mathrm{s}}$ and $\mathrm{X}_{\mathrm{c}}$ are two normalized variables given by

$$
\begin{aligned}
& a_{s}=\frac{\varepsilon_{s}}{\varepsilon_{f}}, \quad a_{c}=\frac{\varepsilon_{c}}{\varepsilon_{f}} \\
& X_{s}=\sqrt{\frac{N^{2}-\varepsilon_{s}}{\varepsilon_{f}-N^{2}}}, X_{s}=\sqrt{\frac{N^{2}-\varepsilon_{s}}{\varepsilon_{f}-N^{2}}}
\end{aligned}
$$

$\mathrm{k}_{0}$ is the free space wave number, $C=k_{0} \sqrt{N^{2}-\varepsilon_{c}}\left(h-z_{c}\right), \mathrm{z}_{\mathrm{c}}$ is a constant related to the field distribution in the covering medium, i.e., the field peaks at $\mathrm{z}=\mathrm{z}_{\mathrm{c}}{ }^{(14-15)}$, and $\mathrm{q}_{\mathrm{f}}$ is given by,

$$
q_{f}=\sqrt{\varepsilon_{f}-N^{2}}=\sqrt{\varepsilon_{f}} \sqrt{\frac{1-a_{s}}{1+X_{s}^{2}}}
$$

$\mathrm{X}_{\mathrm{s}}$ and $\mathrm{X}_{\mathrm{c}}$ are linked by the asymmetry parameters as

$$
X_{c}^{2}=\frac{\left(1-a_{c}\right)\left(1+X_{s}^{2}\right)}{\left(1-a_{s}\right)}-1
$$

The effective refractive index (N) can be written in terms of $a_{s}$ and $\mathrm{X}_{\mathrm{s}}$ as

$$
N=\sqrt{\varepsilon_{f}} \sqrt{\frac{a_{s}+X_{s}^{2}}{1+X_{s}^{2}}}
$$

For sensing purposes, the optical wave-guide structure has to be designed to assure a high sensitivity, that is the sensor response for changes

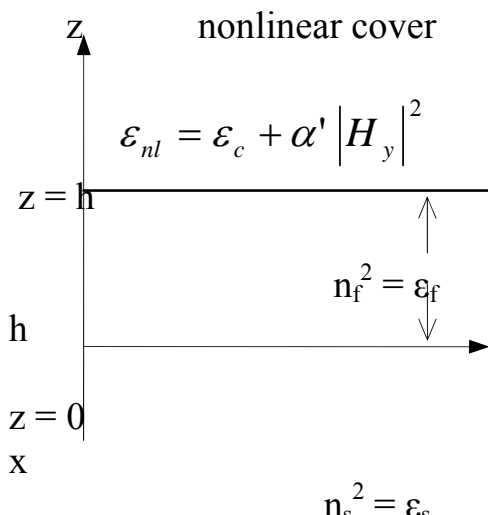

Fig. 1: waveguide configuration 
in the optical properties of the cover medium has to be as high as possible. The sensitivity depends on the strength and distribution of the evanescent field in the cladding ${ }^{(16)}$. In the next step, we will evaluate the sensitivity of the nonlinear sensor when the change in the refractive index of the clad is homogeneously distributed in the cover. In this case, the sensitivity is evaluated as the rate of change of the effective refractive index of the guided mode under an index change of the cover $\left(S_{h}=\partial N / \partial n_{c}\right)$. A partial derivative of the characteristic equations, Eqs. (2), and some algebraic calculations using Eqs. (4-7) will give the following expressions for the homogeneous sensitivity

$$
S_{h}=\frac{\sqrt{a_{c}} \sqrt{1+X_{c}^{2}}\left(a_{c} H+a_{c} \tanh C+2 X_{c}^{2} \tanh C \frac{1-a_{c}}{1+X_{c}^{2}}\right)}{X_{c} \sqrt{a_{c}+X_{c}^{2}} \theta\left(D_{T M}+F_{\text {STM }}+G_{c T M}\right)},
$$

$$
\begin{aligned}
& \text { for } N^{2}<\varepsilon_{f} \\
& S_{h}=\frac{\sqrt{a_{c}} \sqrt{1+X_{c}^{2}}\left(a_{c} H+a_{c} \tanh C+2 X_{c}^{2} \tanh C \frac{1-a_{c}}{1+X_{c}^{2}}\right)}{X_{c} \sqrt{a_{c}+X_{c}^{2}} \theta^{\prime}\left(-D_{T M}^{\prime}+\frac{F_{\text {STM }}\left(a_{s}^{2}+X_{s}^{2}\right)}{\left(a_{s}^{2}-X_{s}^{2}\right)}+\frac{G_{c T M} \theta}{\theta^{\prime}}\right)},
\end{aligned}
$$

$$
\text { for } N^{2}>\varepsilon_{f}
$$

where

$$
\begin{aligned}
& H=k_{0}\left(h-z_{c}\right) X_{c} \sqrt{\varepsilon_{f}} \sqrt{\frac{1-a_{c}}{1+X_{c}^{2}}}\left(1-\tanh ^{2} C\right) \\
& \theta=a_{c}^{2}+X_{c}^{2} \tanh ^{2} C, \theta^{\prime}=a_{c}^{2}-X_{c}^{2} \tanh ^{2} C
\end{aligned}
$$




$$
\begin{gathered}
D_{T M}=\tan ^{-1}\left(\frac{X_{s}}{a_{s}}\right)+\tan ^{-1}\left(\frac{X_{c}}{a_{c}} \tanh C\right)+m \pi, \\
D_{T M}^{\prime}=-\tanh ^{-1}\left(\frac{X_{s}}{a_{s}}\right)-\tanh ^{-1}\left(\frac{X_{c}}{a_{c}} \tanh C\right) \\
F_{\text {STM }}=\frac{a_{s}\left(1+X_{s}^{2}\right)}{X_{s}\left(a_{s}^{2}+X_{s}^{2}\right)} \\
G_{C T M}=\frac{a_{c} H+a_{c} \tanh C\left(1+X_{c}^{2}\right)}{X_{c}\left(a_{c}^{2}+X_{c}^{2} \tanh ^{2} C\right)}
\end{gathered}
$$

where $m$ is the mode order.

One of the most important aims of this work is to provide the designer with the optimum dimensions of the proposed sensor so that the sensor will exhibit its maximum sensing sensitivity. The condition of maximum sensitivity in a structure of constant $\varepsilon_{\mathrm{f}}, \varepsilon_{\mathrm{s}}$ and $\varepsilon_{\mathrm{c}}$ is achieved by cancellation of the derivative of $S_{h}$ with respect to the normalized width $\mathrm{k}_{\mathrm{o}} \mathrm{h}$. That is: $\partial S_{h} / \partial\left(k_{0} h\right)=\left(\partial S_{h} / \partial X_{s}\right) \cdot\left(\partial X_{s} / \partial\left(k_{0} h\right)=0\right.$ and $\partial X_{s} / \partial\left(k_{0} h\right) \neq 0$, so that the condition of maximum sensitivity is attained if the derivative of $S_{h}$ with respect to $X_{s}$ vanishes. Applying this to equations (8) we get 


$$
\begin{aligned}
& X_{c} \theta \sqrt{a_{c}+X_{c}^{2}}\left(D_{T M}+F_{S T M}+G_{c T M}\right) . \\
& \sqrt{a_{c}}\left[\sqrt{1+X_{c}^{2}} U+\left(a_{c} H+a_{c} \tanh C+2 X_{c}^{2} \tanh C \frac{1-a_{c}}{1+X_{c}^{2}}\right) \frac{w X_{s}}{\sqrt{1+X_{c}^{2}}}\right]- \\
& \sqrt{a_{c}} \sqrt{1+X_{c}^{2}}\left(a_{c} H+a_{c} \tanh C+2 X_{c}^{2} \tanh C \frac{1-a_{c}}{1+X_{c}^{2}}\right) . \\
& \left\{X_{c} \sqrt{a_{c}+X_{c}^{2}} \theta(M+J+Q)+\left(D_{T M}+F_{s T M}+G_{c T M}\right) .\right. \\
& {\left[\sqrt{a_{c}+X_{c}^{2}}\left(a_{c}^{2} w \frac{X_{s}}{X_{c}}+2 X_{c}^{3} \tanh C \sigma \zeta+3 \tanh ^{2} C w X_{s} X_{c}\right)+\right.} \\
& \left.\left.\frac{w X_{s} X_{c} \theta}{\sqrt{a_{c}+X_{c}^{2}}}\right]\right\}=0, \text { for } N^{2}<\varepsilon_{f}
\end{aligned}
$$

where

$$
\zeta=\frac{C w \frac{X_{s}}{X_{c}^{2}}+\frac{a_{s} X_{c}}{a_{s}^{2}+X_{s}^{2}}+\frac{a_{c} X_{s} w \tanh C}{\theta}+k_{0} z_{c} \sqrt{\varepsilon_{f}} \frac{X_{s} X_{c}}{1+X_{s}^{2}} \sqrt{\frac{1-a_{s}}{1+X_{s}^{2}}}}{1-\frac{a_{c} X_{c}^{2} \sigma}{\theta}}
$$

$Q=\frac{\begin{array}{c}a_{c} X_{c} \theta\left[\zeta \sigma(1-2 C \tanh C)+2 X_{s} w \tanh C+\zeta\left(1+X_{c}^{2}\right) \sigma\right]- \\ {\left[a_{c} H+a_{c} \tanh C\left(1+X_{c}^{2}\right)\right]\left[a_{c}^{2} w \frac{X_{s}}{X_{c}}+3 w X_{c} X_{s} \tanh ^{2} C+2 \zeta \sigma X_{c}^{3} \tanh C\right]}\end{array}}{X_{c}^{2} \theta^{2}}$

$$
\begin{aligned}
& \sigma=1-\tanh ^{2} C \\
& w=\frac{1-a_{c}}{1-a_{s}}
\end{aligned}
$$

$$
U=2 a_{c} \sigma \zeta(1-C \tanh C)+\frac{4\left(1-a_{c}\right) w X_{s} \tanh C}{\left(1+X_{c}^{2}\right)^{2}}+2\left(1-a_{c}\right) \sigma \zeta \frac{X_{c}^{2}}{1+X_{c}^{2}}
$$




$$
\begin{gathered}
J=\frac{2 a_{s} X_{s}^{2}\left(a_{s}^{2}+X_{s}^{2}\right)-a_{s}\left(1+X_{s}^{2}\right)\left(a_{s}^{2}+3 X_{s}^{2}\right)}{X_{s}^{2}\left(a_{s}^{2}+X_{s}^{2}\right)^{2}} \\
M=\frac{a_{s}}{a_{s}^{2}+X_{s}^{2}}+\frac{a_{c} X_{c} \sigma \zeta}{\theta}+\frac{a_{c} \tanh C w X_{s}}{\theta X_{c}}
\end{gathered}
$$

The condition for maximum sensing sensitivity for $\mathrm{N}^{2}>\varepsilon_{\mathrm{f}}$ can be written as,

$$
\begin{aligned}
& X_{c} \theta^{\prime} \sqrt{a_{c}+X_{c}^{2}}\left(-D_{T M}^{\prime}+\frac{F_{S T M}\left(a_{s}^{2}+X_{s}^{2}\right)}{a_{s}^{2}-X_{s}^{2}}+\frac{G_{c T M} \theta}{\theta^{\prime}}\right) . \\
& \sqrt{a_{c}}\left[\sqrt{1+X_{c}^{2}} U^{\prime}+\left(a_{c} H+a_{c} \tanh C+2 X_{c}^{2} \tanh C \frac{1-a_{c}}{1+X_{c}^{2}}\right) \frac{w X_{s}}{\sqrt{1+X_{c}^{2}}}\right]- \\
& \sqrt{a_{c}} \sqrt{1+X_{c}^{2}}\left(a_{c} H+a_{c} \tanh C+2 X_{c}^{2} \tanh C \frac{1-a_{c}}{1+X_{c}^{2}}\right) . \\
& \left\{X_{c} \sqrt{a_{c}+X_{c}^{2}} \theta^{\prime}\left(-M^{\prime}+J^{\prime}+\frac{2 a_{c}^{2} G_{c T M} L+\theta \theta^{\prime} Q^{\prime}}{\theta^{\prime 2}}\right)+\left(-D_{T M}^{\prime}+\frac{F_{s T M}\left(a_{s}^{2}+X_{s}^{2}\right)}{a_{s}^{2}-X_{s}^{2}}+\frac{G_{c T M} \theta}{\theta^{\prime}}\right) .\right. \\
& \left.\left[\sqrt{a_{c}+X_{c}^{2}}\left(a_{c}^{2} w \frac{X_{s}}{X_{c}}-2 X_{c}^{3} \tanh C \sigma \zeta^{\prime}-3 \tanh ^{2} C w X_{s} X_{c}\right)+\frac{w X_{s} X_{c} \theta^{\prime}}{\sqrt{a_{c}+X_{c}^{2}}}\right]\right\}=0
\end{aligned}
$$

Where

$$
\begin{gathered}
\zeta^{\prime}=\frac{C w \frac{X_{s}}{X_{c}^{2}}-\frac{a_{s} X_{c}}{a_{s}^{2}-X_{s}^{2}}-\frac{a_{c} X_{s} w \tanh C}{\theta^{\prime}}+k_{0} z_{c} \sqrt{\varepsilon_{f}} \frac{X_{s} X_{c}}{1+X_{s}^{2}} \sqrt{\frac{1-a_{s}}{1+X_{s}^{2}}}}{1+\frac{a_{c} X_{c}^{2} \sigma}{\theta^{\prime}}} \\
J^{\prime}=\frac{4 a_{s}^{2} F_{s T M} X_{s}+\left(a_{s}^{2}-X_{s}^{2}\right)\left(a_{s}^{2}+X_{s}^{2}\right) J}{\left(a_{s}^{2}-X_{s}^{2}\right)^{2}}
\end{gathered}
$$




$$
\begin{gathered}
M^{\prime}=-\frac{a_{s}}{a_{s}^{2}-X_{s}^{2}}-\frac{a_{c} X_{c} \sigma \zeta^{\prime}}{\theta^{\prime}}-\frac{a_{c} \tanh C w X_{s}}{\theta^{\prime} X_{c}} \\
L=2 X_{c}^{2} \tanh C \sigma \zeta^{\prime}+2 \tanh ^{2} C w X_{s}
\end{gathered}
$$

$\mathrm{Q}^{\prime}$ and $\mathrm{U}^{\prime}$ are same as $\mathrm{Q}$ and $\mathrm{U}$ respectively with $\zeta^{\prime}$ replaces $\zeta$ in Eqs. (16) and (18).

The solution of each of the equations (14) and (21) is one value of the normalized index $X_{s}$ for each pair of the asymmetry parameters $\left(a_{s}, a_{c}\right)$. Substituting the $X_{s}$ solutions into the dispersion equations, we get the values of waveguide thickness (h) needed for the designer so that the proposed sensor will work in the region of maximum sensitivity. It is interesting to locate the optimum value of $h$ with respect to the cut-off of the sensing mode. At cut-off, the effective index $\mathrm{N}$ approaches the substrate index $\mathrm{n}_{\mathrm{s}}$ and the evanescent field in the substrate approaches infinity for the traditional waveguide geometry $\left(\mathrm{n}_{\mathrm{s}}>\mathrm{n}_{\mathrm{c}}\right)$. No power flows in the cladding and the effective index does not feel the changes occur in the index of the cover. The slab waveguide is not a sensor any more at cutoff. The waveguide thickness at cut-off for the fundamental mode can be obtained from expressions (8) with $\mathrm{X}_{\mathrm{s}}=0$,

$$
h_{\text {cut }- \text { off }}=\frac{\tan ^{-1}\left(\frac{\tanh C}{a_{c}} \sqrt{\frac{a_{s}-a_{c}}{1-a_{s}}}\right)}{k_{o} \sqrt{\varepsilon_{f}} \sqrt{1-a_{s}}}, \text { for } N^{2}<\varepsilon_{f}
$$

For $\mathrm{N}^{2}>\varepsilon_{\mathrm{f}}$, - arctanh replaces arctan in Eq. (26) to obtain the cut-off thickness.

It is essential when speaking about optical sensing to consider the power flow in the waveguide layers. The sensitivity of an optical sensor is critically dependent on the fraction of power 
propagating in the covering medium. Enhancement of the sensitivity requires getting as much of the optical power as possible to propagate in the cover. For TM modes, the time averaged power flow in the $\mathrm{x}$ direction per unit width in the $\mathrm{y}$ direction can be expressed as:

$$
P=\frac{N k_{o}}{2 \omega \varepsilon_{o} \varepsilon_{r}} \int_{-\infty}^{\infty} H_{y}^{2} d z=P_{s}+P_{f}+P_{c}
$$

The power fraction in the covering medium can be written as

$$
\begin{aligned}
\frac{P_{c}}{P_{\text {total }}}= & \frac{\frac{X_{c} q_{f}}{\alpha^{\prime} a_{c}}(1-\tanh C)}{\frac{X_{c} q_{f}}{\alpha^{\prime} a_{c}}(1-\tanh C)+\frac{a_{2}^{2}}{4 q_{f}}\left[k_{o} q_{f} h\left(1+\frac{X_{s}^{2}}{a_{s}^{2}}\right)+\frac{1}{2} \sin \left(2 k_{o} q_{f} h\right)\left(1-\frac{X_{s}^{2}}{a_{s}^{2}}\right)+\right.} \\
& \left.\frac{X_{s}}{a_{s}}\left(1-\cos \left(2 k_{o} q_{f} h\right)\right)\right]+\frac{a_{2}^{2}}{4 X_{s} a_{s} q_{f}}, \text { for } N^{2}<\varepsilon_{f}
\end{aligned}
$$

where

$$
a_{2}=\frac{\sqrt{2} X_{c} q_{f}}{\sqrt{\alpha^{\prime}} \cosh C\left(\cos \left(k_{o} q_{f} h\right)+\frac{X_{s}}{a_{s}} \sin \left(k_{o} q_{f} h\right)\right)}
$$

For $\mathrm{N}^{2}>\varepsilon_{\mathrm{f}}$, one needs to replace $\sin \left(2 \mathrm{k}_{\mathrm{o}} \mathrm{q}_{\mathrm{f}} \mathrm{h}\right), \frac{X_{s}^{2}}{a_{s}^{2}}$, and $[1-$ $\left.\cos \left(2 \mathrm{k}_{\mathrm{o}} \mathrm{q}_{\mathrm{f}} \mathrm{h}\right)\right]$ by $\sinh \left(2 \mathrm{k}_{\mathrm{o}} \mathrm{q}_{\mathrm{f}} \mathrm{h}\right),-\frac{X_{\mathrm{s}}^{2}}{a_{\mathrm{s}}^{2}}$, and $\left[\cosh \left(2 \mathrm{k}_{\mathrm{o}} \mathrm{q}_{\mathrm{f}} \mathrm{h}\right)-1\right]$ respectively in expression (28) and to replce $\sin \left(\mathrm{k}_{\mathrm{o}} \mathrm{q}_{\mathrm{f}} \mathrm{h}\right)$ and $\cos \left(\mathrm{k}_{\mathrm{o}} \mathrm{q}_{\mathrm{f}} \mathrm{h}\right)$ by $\sinh \left(\mathrm{k}_{\mathrm{o}} \mathrm{q}_{\mathrm{f}} \mathrm{h}\right)$ and $\cosh \left(\mathrm{k}_{\mathrm{o}} \mathrm{q}_{\mathrm{f}} \mathrm{h}\right)$ respectively in expression (29). 


\section{Results AND DISCUSSION:}

The formalism above enables the designer to find the required parameters to construct an optical sensor for $\mathrm{p}$ polarized waves and to choose the optimum parameters ensuring maximum sensitivity. The procedure to retrieve these parameters and to find the maximum sensitivity that can be achieved is as follows. The condition required for the sensor to exhibit its maximum sensing sensitivity is given by Eq. (14) for $\varepsilon_{\mathrm{f}}>\mathrm{N}^{2}$ and by Eq. (21) for $\varepsilon_{\mathrm{f}}<\mathrm{N}^{2}$. Given the asymmetry parameters $\left(a_{s}, a_{c}\right)$ and making use of Eq. (6) to substitute for $X_{c}$ in terms of $X_{s}$, Eq. (14) and Eq. (21) become functions of $X_{s}$ only. The solution to each of them is one value of the normalized refractive index $X_{s}$ for each pair of the asymmetry parameters. The effective refractive index $\mathrm{N}$ corresponding to the optimum value of $X_{s}$ is given by expression (7). The thickness of the waveguide ensuring maximum sensitivity can be found by substituting the optimum value of $X_{s}$ into Eq. (8a) for $\varepsilon_{\mathrm{f}}>\mathrm{N}^{2}$ and into Eq. (8b) for $\varepsilon_{\mathrm{f}}<\mathrm{N}^{2}$.

The numerical calculations were performed with $\mathrm{n}_{\mathrm{f}}=$ $2, \lambda=1550 \mathrm{~nm}$ and tanhc $=0.6$. We will restrict ourselves to the fundamental mode $(\mathrm{m}=0)$, which is most often the case because it has the highest sensitivity compared with higher modes ${ }^{(11)}$. However, our analytical expressions are valid for any mode order $\mathrm{m}$. The present work is limited to conventional symmetry, a configuration for which $n_{s}>n_{c}$, except in fig. (7), we investigated the behavior of the sensor in case of reverse symmetry.

The sensitivity of the proposed sensor was plotted with the asymmetry parameters $a_{c}$ and $a_{s}$ in Fig. 2 and in Fig. 3 respectively. The figures show that the sensitivity increases with increasing $a_{c}$ and decreases with increasing $a_{s}$. This is a normal behavior since the sensing operation is performed by the evanescent optical field extending from the thin guiding film into the covering medium. To obtain high sensitivity, it is 
essential to get as much of the optical power as possible to propagate in the cover. In this sense, increasing $a_{c}$ and decreasing $a_{\mathrm{s}}$ will enhance the power fraction flowing in the cladding medium. A comparison between linear sensors ( $\operatorname{tanhC}=1$ in expressions 8$)$, and nonlinear sensors is also shown in Fig. 2 and Fig. 3. The nonlinear sensitivities are shown to have higher values than the corresponding linear sensitivities when the effective index is less than the index of the guiding layer. Fig. 4 shows the sensitivity as a function of the slab waveguide thickness for two values of $a_{c}$. The sensitivity has a peak at a specific value of $h$, which we call the optimum value oh $\mathrm{h}$, and this peak is shifted towards lower values of $h$ and higher values of the sensitivity with increasing $a_{c}$. At small values of $h$ near cut-off, the sensitivity approaches zero since the effective refractive index approaches the index of the substrate and the fraction of power flowing in the clad vanishes. To the right of this peak, we can see the sensitivity increases as the core thickness diminishes due to a less confinement of light within the core, which implies an increase of the power of the guided mode transported in the cover medium. At relatively very high values of $h$, the sensitivity again approaches zero due to the high confinement of light within the core. For the sake of comparison, the sensitivity was plotted with $\mathrm{h}$ in Fig. 5 for $\mathrm{N}<\mathrm{n}_{\mathrm{f}}, \mathrm{N}>\mathrm{n}_{\mathrm{f}}$ and for the linear case. Again the nonlinear sensor with effective refractive index less than the film index has higher sensitivities. To show the behavior of the sensor for different light polarization, we plotted the sensitivity with the core thickness for $\mathrm{TM}_{\mathrm{o}}$ and $\mathrm{TE}_{\mathrm{o}}$ modes in Fig. 6. We see that the sensitivity in $\mathrm{TM}_{\mathrm{o}}$ has higher values at thicker thicknesses. Therefore, TM configuration is recommended. In Fig. 7 we demonstrate a reverse symmetry waveguide sensor $\left(\mathrm{n}_{\mathrm{s}}<\mathrm{n}_{\mathrm{c}}\right)$, which exhibits much higher sensitivity than a conventional waveguide sensor. The reason of this behavior is the power considerations. In principle, this configuration (reverse 
symmetry) allows most of the optical power to present in the covering medium which is contrary to the conventional waveguide geometry, where typically less than $10 \%$ of the total power is present in the cladding medium. The configuration considered in this work is the normal symmetry since it is the case of most practical cases and our interest here is dedicated to the effect of nonlinearity on the sensitivity. Fig. 8 demonstrates the effect of light wavelength on the performance of the sensor. As the wavelength increases, the core thickness corresponding to the maximum sensitivity increases whereas maximum value of the sensitivity doesn't change with $\lambda$. Thus $\lambda$ is a determinant parameter for the thickness at which the maximum sensitivity occurs. The close connection between the sensitivity of the proposed sensor and the fraction of power flowing in the cover is shown in Fig. 9 and Fig. 10. Their behavior is almost identical thus the enhancement of the fraction of power flowing in the clad is essential for sensing applications. The variation of the sensitivity with the term tanhC, which arises from the nonlinearity of the clad, is plotted in Fig. 11. As the term tanhC goes to one in expressions (8), we obtain the well known characteristic equations for linear waveguides. The figure shows the sensitivity to have the minimum value as tanhC goes to one. We believe that nonlinear sensors have higher sensitivities than linear one's and can improve the performance of optical sensors. Finally, we present the procedure that should be followed by the designer to construct the proposed sensor with the optimum parameters. Expressions (14) and (21) contain the condition required for the sensor to exhibit it's maximum sensing sensitivity. Plotting these expressions gives the surface $X_{s}\left(a_{s}, a_{c}\right)$ in Fig. 12 for $N$ $<\mathrm{n}_{\mathrm{f}}$ and the surface $\mathrm{X}_{\mathrm{s}}\left(\mathrm{a}_{\mathrm{s}}, \mathrm{a}_{\mathrm{c}}\right)$ in Fig. 13 for $\mathrm{N}>\mathrm{n}_{\mathrm{f}}$. All the necessary information to construct the optimized sensor is contained in the surface $X_{s}\left(a_{s}, a_{c}\right)$ and the above set of equations. A typical way of proceeding is as follow. Usually 
the choice of the cover material is determined by the sensor usage, thus its nominal index $n_{c}$. The substrate material and its refractive index $n_{s}$ result from a choice taking into consideration temperature, mechanical stability and cost criteria. The optical and chemical stability are the criteria considered in the choice of the guiding layer material. Once the refractive indices of the three layers and the asymmetry parameters $a_{s}$ and $a_{c}$ are determined, the designer should refer to Fig. 12 for $\mathrm{N}<\mathrm{n}_{\mathrm{f}}$ or to Fig. 13 for $\mathrm{N}>\mathrm{n}_{\mathrm{f}}$ to find the optimum value of $X_{s}$ which provides the highest sensitivity. The optimum values of the normalized variable $X_{c}$, effective index $\mathrm{N}$, and the guiding layer thickness $\mathrm{h}$ are obtained by substituting the optimum value of $\mathrm{X}_{\mathrm{s}}$ into Eq. (6), Eq. (7) and Eq. (2) respectively. The maximum achievable sensitivity is obtained by substituting these values into expressions (8). To clarify this procedure, we consider the following example for $\mathrm{N}<\mathrm{n}_{\mathrm{f}}$. Let us consider $\mathrm{a}_{\mathrm{s}}=0.31$ and $\mathrm{a}_{\mathrm{c}}=0.3$. The solution of Eq. (14), Fig. (12), gives $X_{\mathrm{s}}$ (optimum) $=0.3872$. Introducing this value into Eq. (2a) with the help of Eq. (6) and Eq. (7) yields the optimum value of the film thickness, $h$ (optimum) $=$ $0.265 \mu \mathrm{m}$. Making use of Eq. (8a), the maximum achievable sensitivity is found to be $\mathrm{S}_{\max }=0.55$. This is the highest sensitivity for the proposed values of refractive indices. The cut-off thickness was also calculated using Eq. (26) and the result was $h_{\text {cut-off }}=0.0417 \mu \mathrm{m}$. In ${ }^{(15)}$, we presented the same work for TE modes. For the same values of $a_{s}$ and $a_{c}$, we calculated the optimum parameters for $\mathrm{TE}_{\mathrm{o}}$ mode. The results were $\mathrm{X}_{\mathrm{s}}($ optimum $)=0.2127, \mathrm{~h}($ optimum $)=0.06 \mu \mathrm{m}, \mathrm{S}_{\max }=$ 0.437 and $h_{\text {cut-off }}=0.013 \mu \mathrm{m}$. Inspection of these results reveals that for TE modes, the configurations exhibiting the maximum sensitivity correspond to the proximity of the cutoff whereas for TM modes, the configurations of maximum sensitivity correspond to larger distances from cut-off. Moreover, the maximum sensitivity for TM modes has higher 
values. In most practical cases, it is advantageous to use $\mathrm{TM}_{\mathrm{o}}$ modes.

\section{CONCLUSION:}

In this paper, a nonlinear slab waveguide was analyzed as an optical sensor. The sensitivity of the proposed sensor was studied with various parameters of the structure. The procedure to get the highest output of the sensor was shown. In conclusion, we emphasize that nonlinear sensors can improve the performance of optical sensors. We recommend TM modes with the effective refractive index less than the index of the core medium. 


\section{FIGURES:}

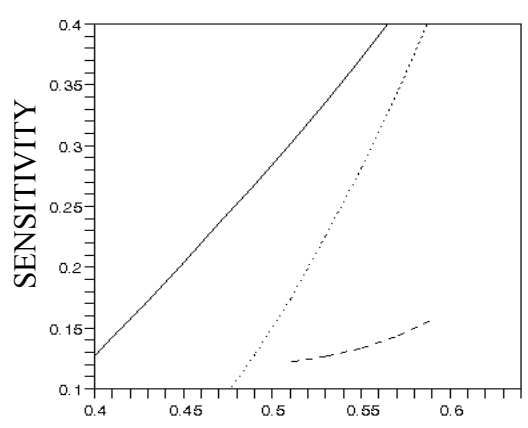

$\mathrm{a}_{\mathrm{c}}$

FIG. 2. Sensitivity as a function of the asymmetry parameter $a_{\mathrm{c}}$ for $\mathrm{a}_{\mathrm{s}}=0.6$ and $\mathrm{h}=0.2 \mu \mathrm{m}$ for three different cases, $\mathrm{N}<$ $\mathrm{n}_{\mathrm{f}}$ (solid line), $\mathrm{N}>\mathrm{n}_{\mathrm{f}}$ (dashed line) and a sensor with a linear clad (dotted line).

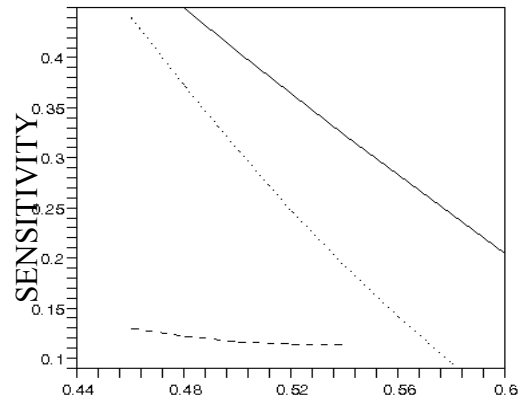

$a_{s}$

FIG. 3. Sensitivity as a function of the asymmetry parameter $a_{\mathrm{s}}$ for $\mathrm{a}_{\mathrm{c}}=0.45$ and $\mathrm{h}=0.2 \mu \mathrm{m}$ for three different cases, $\mathrm{N}<\mathrm{n}_{\mathrm{f}}$ (solid line), $\mathrm{N}>\mathrm{n}_{\mathrm{f}}$ (dashed line) and a sensor with a linear clad (dotted line).

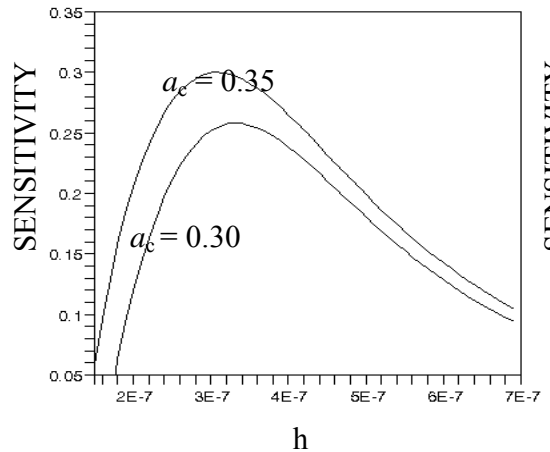

FIG. 4. Sensitivity with the guiding layer thickness for $\mathrm{a}_{\mathrm{s}}=0.51 .\left(\mathrm{N}<\mathrm{n}_{\mathrm{f}}\right)$

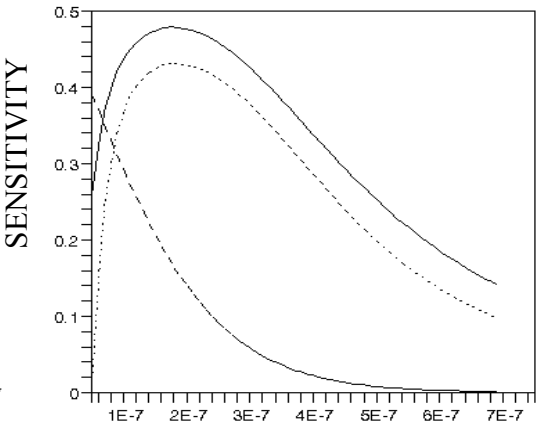

$\mathrm{h}$

FIG. 5. Sensitivity with the film thickness for $\mathrm{a}_{\mathrm{s}}=0.51$ and $\mathrm{a}_{\mathrm{c}}=0.5$ for three different cases, $\mathrm{N}<\mathrm{n}_{\mathrm{f}}$ (solid line), $\mathrm{N}>\mathrm{n}_{\mathrm{f}}$ (dashed line) and a linear sensor (dotted line). 


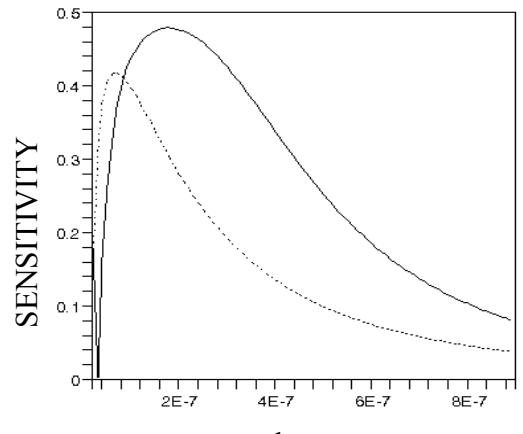

$\mathrm{h}$

FIG. 6. Sensitivity as a function of waveguide film thickness for $\mathrm{TM}_{\mathrm{o}}$ mode (solid line) and for $\mathrm{TE}_{\mathrm{o}}$ modes (dotted line). $\mathrm{a}_{\mathrm{s}}=0.51$ and $\mathrm{ac}=0.5$. $(\mathrm{N}$ $\left.<\mathrm{n}_{\mathrm{f}}\right)$

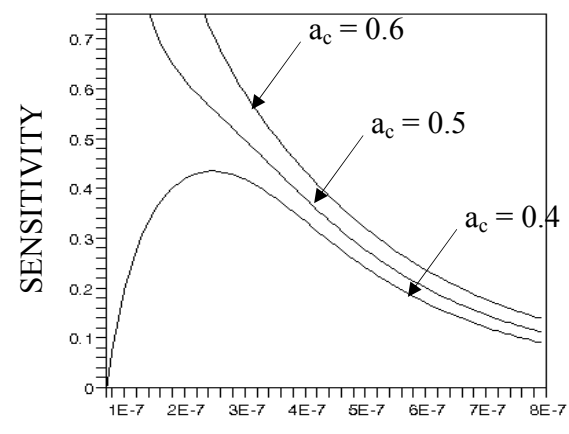

$\mathrm{h}$

FIG. 7. Sensitivity as a function of waveguide film thickness for a waveguide with normal symmetry $\left(\mathrm{a}_{\mathrm{s}}>\right.$ $\mathrm{a}_{\mathrm{c}}$ ) and a waveguide with reverse symmetry $\left(a_{\mathrm{s}}<\mathrm{a}_{\mathrm{c}}\right) . \mathrm{a}_{\mathrm{s}}=0.45$ for all cases. $\left(\mathrm{N}<\mathrm{n}_{\mathrm{f}}\right)$

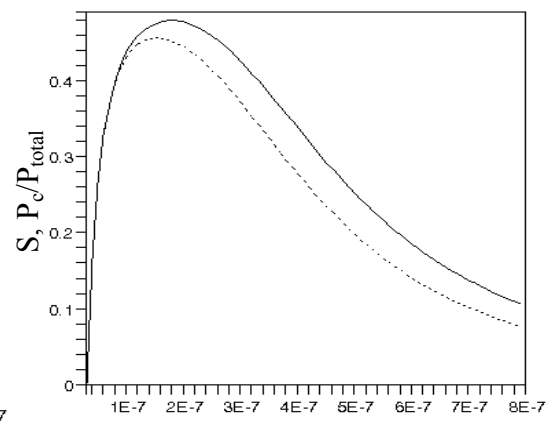

$\mathrm{h}$

FIG. 9. The sensitivity (solid line) and fraction of total power propagating in the cover medium (dotted line) as a function of the film thickness. $\left(a_{c}=0.5\right.$ $\left.\mathrm{a}_{\mathrm{s}}=0.51, \alpha^{\prime}=66.44 \times 10^{-13}, \mathrm{~N}<\mathrm{n}_{\mathrm{f}}\right)$ 


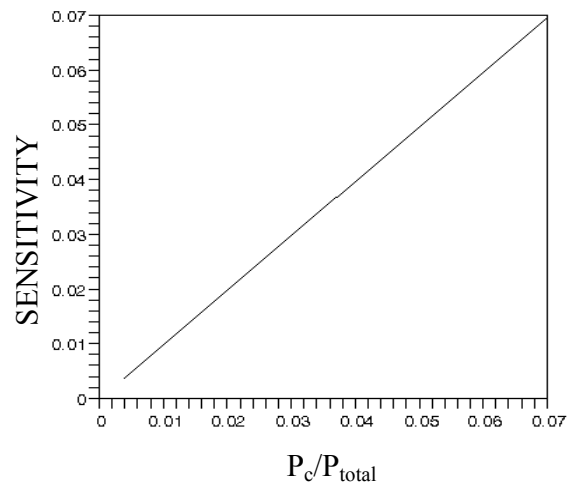

FIG. 10. Sensitivity against the fraction of total power propagating in the cover medium. $\left(\mathrm{a}_{\mathrm{c}}=0.5, \mathrm{a}_{\mathrm{s}}=0.51\right.$, $\left.\alpha^{\prime}=66.44 \times 10^{-13}, \mathrm{~N}<\mathrm{n}_{\mathrm{f}}\right)$

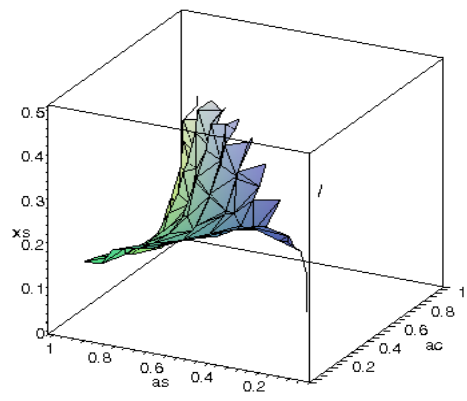

FIG. 12. Normalized effective refractive index $X_{s}$ versus $a_{s}$ and $a_{c}$ ensuring maximum sensitivity for homogeneous sensing for $\mathrm{N}<\mathrm{n}_{\mathrm{f}}$.

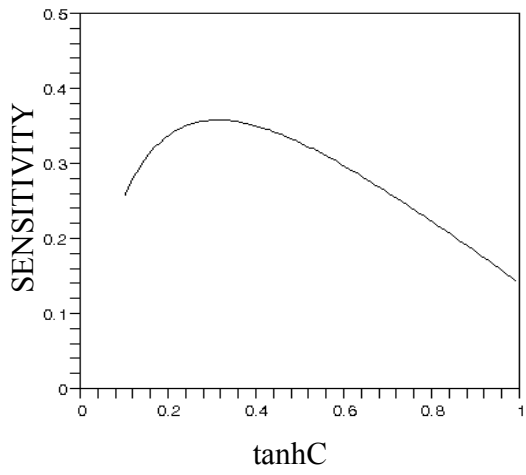

FIG. 11. Sensitivity against tanhC for $\mathrm{a}_{\mathrm{c}}$ $=0.3 \mathrm{a}_{\mathrm{s}}=0.41$, and $\mathrm{h}=0.2 \mu \mathrm{m} .(\mathrm{N}<$ $\mathrm{n}_{\mathrm{f}}$.

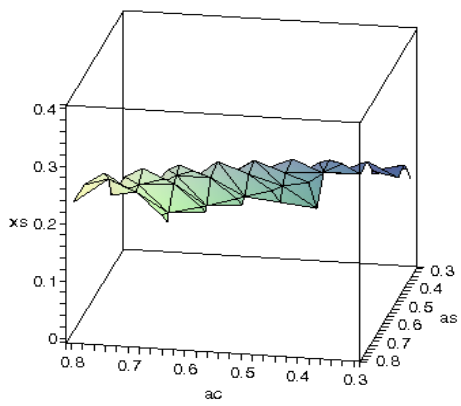

FIG. 13. Normalized effective refractive index $X_{s}$ versus $a_{s}$ and $a_{c}$ ensuring maximum sensitivity for homogeneous sensing for $\left(\mathrm{N}>\mathrm{n}_{\mathrm{f}}\right)$.. 


\section{REFERENCES:}

1. K. Benaissa, A. Nathan: Silicon anti-resonant reflecting optical waveguides for sensor applications. Sensors and Actuators A 65 (1998) 33-44

2. R. G. C. Oudshoorn, R. P. H. Kooyman J. Greve: Refractive index and layer thickness of an adsorbing protein as reporters of monolayer formation. Thin solid films 284-285 (1996) 836-840

3. D. K. Qing, X. M. Chen, K. Itoh M. Murabayashi: A theoretical evaluation of the absorption coefficient of the optical waveguide chemical or biological sensors by group index method. J. of Lightwave Tech 14, 8 (1996) 1907-1917

4. S. Plunkett, S. Propst M. Braiman: Supported planar germanium waveguides of infrared evanescent-wave sensing. App. Optics 36, 18 (1997) 4055-4061

5. M. M. Abadla M. M. Shabat: Characteristics of nonlinear waveguides sensors with metallic core films", J. Laser Phys. 14, 12 (2004) 1524-1528

6. G. J. Veldhuis, O. Parriaux, H. J. W. M. Hoekstra, P. V. Lambeck: Sensitivity enhancement in evanescent optical waveguide sensor. J. of Lightwave Tech. 18, 5 (2000) 667-682

7. O. Parriaux, P. V. Lambeck, H. J. W. M. Hoekstra, G. J. Veldhuis, G. Pandraud: Evanescent wave sensor of sensitivity larger than a free space wave. Opt. and Quantum Electronics 32 (2000) 909-921

8. G. J. Veldhuis, O. Parriaux, P. V. Lambeck: Normalized analysis for the optimization of geometric wavelength dispersion in three-layer slab waveguides. Opt. Communication 163 (1999) 278-284

9. N. Skivesen, R. Horvath, H. Pedersen: Multimode reverse-symmetry waveguide sensor for broad-range refractometry. Opt. Letters 28, 24 (2003) 2473-2475

10. R. Horvath, L. R. Lindvold, N. B. Larsen: Fabrication of all polymer freestanding waveguides. 13 (2003) 419-424

11. O. Parriaux, G. J. Velduis: Normailzed analysis for the sensitivity optimization of integrated optics evanescent-wave sensors. J. of Lightwave Tech. 16, 4 (1998) 573-582

12. M.M.Shabat, "TM non-linear electromagnetic waves guided by an inhomogeneous dielectric medium", International Journal of Infrared and Millimetre Waves, vol.14(5), (1993) 1107-1115

13. A.D.Boardman, M.M.Shabat, and R.F.Wallis, "'Nonlinear surface magnetoplasmons waves on semiconductors", Opt.Comm. vol.86, (1991) 614-622

14. C. Xuelong, H. Zhaoming, Z. Yowei: Spatial bistability in nonlinear optical waveguides. Chinese Phys.-Lasers 15, 5 (1988) 381-383

15. S. Chelkowski J. Chrostowski: Scaling rules for slab waveguides with nonlinear substrate. Applied Optics 26, 17 (1987) 3681-3688

16. W. Lukosz: Principles and sensitivities of integrated optical and surface plasmon sensors for direct affinity sensing and immunosensing. Biosen. Bioelectron. 6 (1991) 215-225 
17. M.Abadla, M.M.Shabat, and D.Jäger, Simulation of sensitivity characteristics in optical nonlinear wave guide sensors, Laser Physics, Vol.14, No.9, (2004)

1231-1237. 